\title{
Page S1
}

\section{Supporting Information}

Reference (31): M. J. Frisch, G. W. T., H. B. Schlegel, G. E. Scuseria, ; M. A. Robb, J. R. C., J. A. Montgomery, Jr., T. Vreven, ; K. N. Kudin, J. C. B., J. M. Millam, S. S. Iyengar, J. Tomasi, ; V. Barone, B. M., M. Cossi, G. Scalmani, N. Rega, ; G. A. Petersson, H. N., M. Hada, M. Ehara, K. Toyota, ; R. Fukuda, J. H., M. Ishida, T. Nakajima, Y. Honda, O. Kitao, ; H. Nakai, M. K., X. Li, J. E. Knox, H. P. Hratchian, J. B. Cross, ; C. Adamo, J. J., R. Gomperts, R. E. Stratmann, O. Yazyev, ; A. J. Austin, R. C., C. Pomelli, J. W. Ochterski, P. Y. Ayala, ; K. Morokuma, G. A. V., P. Salvador, J. J. Dannenberg, ; V. G. Zakrzewski, S. D., A. D. Daniels, M. C. Strain, ; O. Farkas, D. K. M., A. D. Rabuck, K. Raghavachari, ; J. B. Foresman, J. V. O., Q. Cui, A. G. Baboul, S. Clifford, ; J. Cioslowski, B. B. S., G. Liu, A. Liashenko, P. Piskorz, ; I. Komaromi, R. L. M., D. J. Fox, T. Keith, M. A. Al-Laham, ; C. Y. Peng, A. N., M. Challacombe, P. M. W. Gill, ; B. Johnson, W. C., M. W. Wong, C. Gonzalez, and J. A. Pople Gaussian 03W, RevC.02, Gaussian, Inc., Wallingford, CT 064922004. 
Page S2

Table S1 Percentage Composition of N945H2 Frontier Molecular Orbitals (INDO/S)

\begin{tabular}{|c|c|c|c|c|c|}
\hline$\# \mathrm{MO}$ & Energy $/ \mathrm{eV}$ & $\% \mathrm{CO}_{2}$ bpy & $\% \mathrm{Ru}$ & $\% \mathrm{NCS}$ & $\%$ Styrylbpy \\
\hline 162 & -0.23 & 1.1 & 0.6 & 0.1 & 98.2 \\
\hline 161 & -0.82 & 2.9 & 0.0 & 0.0 & 97.0 \\
\hline 160 & -0.94 & 96.9 & 0.1 & 0.0 & 3.0 \\
\hline 159 & -1.36 & 0.4 & 1.2 & 0.1 & 98.3 \\
\hline 158 & -1.62 & 89.5 & 2.8 & 0.2 & 7.5 \\
\hline 157 & -1.75 & 14.4 & 5.2 & 0.7 & 79.8 \\
\hline $\mathbf{1 5 6 L}$ & -2.09 & 82.6 & 7.3 & 1.1 & 9.1 \\
\hline \hline $\mathbf{1 5 5 H}$ & -6.81 & 7.7 & 37.5 & 49.7 & 5.2 \\
\hline 154 & -7.05 & 6.7 & 33.1 & 59.0 & 1.2 \\
\hline 153 & -7.08 & 1.2 & 36.1 & 56.9 & 5.9 \\
\hline 152 & -7.51 & 0.2 & 0.1 & 99.4 & 0.3 \\
\hline 151 & -8.00 & 1.1 & 21.9 & 37.5 & 39.5 \\
\hline 150 & -8.13 & 3.8 & 15.8 & 39.0 & 41.4 \\
\hline 149 & -8.17 & 11.0 & 41.3 & 45.3 & 2.4 \\
\hline 148 & -8.44 & 3.7 & 14.1 & 17.9 & 64.2 \\
\hline
\end{tabular}


Page S3

\begin{tabular}{|c|c|c|c|c|c|}
\hline \multicolumn{6}{|c|}{ Table S2 S1 Percentage Composition of N945H Frontier Molecular Orbitals (INDO/S) } \\
\hline$\#$ MO & Energy /eV & $\% \mathrm{CO}_{2}$ bpy & $\% \mathrm{Ru}$ & $\%$ NCS & \%Styrylbpy \\
\hline 161 & 1.36 & 0.7 & 0.8 & 0.1 & 98.4 \\
\hline 160 & 1.04 & 98.4 & 0.8 & 0.1 & 0.7 \\
\hline 159 & 0.77 & 0.4 & 0.4 & 0.1 & 99.0 \\
\hline 158 & 0.18 & 6.4 & 2.1 & 0.2 & 91.3 \\
\hline 157 & 0.09 & 58.1 & 7.5 & 1.0 & 33.4 \\
\hline $\mathbf{1 5 6 L}$ & -0.16 & 29.2 & 2.2 & 0.3 & 68.3 \\
\hline \hline $\mathbf{1 5 5 H}$ & -4.89 & 7.1 & 42.8 & 42.7 & 7.4 \\
\hline 154 & -5.11 & 7.5 & 44.7 & 46.1 & 1.7 \\
\hline 153 & -5.18 & 1.7 & 43.8 & 48.0 & 6.6 \\
\hline 152 & -5.70 & 0.2 & 0.1 & 99.5 & 0.2 \\
\hline 151 & -5.80 & 99.6 & 0.2 & 0.1 & 0.1 \\
\hline 150 & -6.01 & 100.0 & 0.0 & 0.0 & 0.0 \\
\hline 149 & -6.16 & 4.5 & 26.2 & 53.2 & 16.1 \\
\hline 148 & -6.21 & 12.9 & 28.6 & 57.3 & 1.2 \\
\hline 147 & -6.29 & 95.6 & 1.0 & 2.8 & 0.7 \\
\hline
\end{tabular}


Page S4

\begin{tabular}{|c|c|c|c|c|c|}
\hline \multicolumn{6}{|c|}{ Table S3 Percentage Composition of N945 Frontier Molecular Orbitals (INDO/S) } \\
\hline \#MO & Energy /eV & $\% \mathrm{CO}_{2}$ bpy & $\% \mathrm{Ru}$ & $\% \mathrm{NCS}$ & \%Styrylbpy \\
\hline 162 & 3.07 & 71.2 & 1.9 & 0.5 & 26.4 \\
\hline 161 & 2.99 & 0.4 & 0.8 & 0.1 & 98.7 \\
\hline 160 & 2.89 & 0.0 & 0.1 & 0.0 & 99.9 \\
\hline 159 & 2.86 & 0.0 & 0.0 & 0.0 & 100.0 \\
\hline 158 & 2.44 & 0.6 & 1.7 & 0.2 & 97.6 \\
\hline 157 & 1.76 & 0.0 & 1.2 & 0.1 & 98.6 \\
\hline $\mathbf{1 5 6 L}$ & 1.64 & 0.8 & 6.6 & 0.8 & 91.8 \\
\hline $\mathbf{1 5 5 H}$ & -2.99 & 5.6 & 48.8 & 34.4 & 11.1 \\
\hline 154 & -3.19 & 8.4 & 51.3 & 38.7 & 1.7 \\
\hline 153 & -3.34 & 2.0 & 55.6 & 31.9 & 10.6 \\
\hline 152 & -3.97 & 1.4 & 1.3 & 97.2 & 0.2 \\
\hline 151 & -4.00 & 99.5 & 0.1 & 0.3 & 0.0 \\
\hline 150 & -4.16 & 99.7 & 0.0 & 0.2 & 0.1 \\
\hline 149 & -4.20 & 100.0 & 0.0 & 0.0 & 0.0 \\
\hline 148 & -4.29 & 19.8 & 19.4 & 60.0 & 0.8 \\
\hline
\end{tabular}


Page S5

\begin{tabular}{|c|c|c|c|c|c|}
\hline \multicolumn{6}{|c|}{$\begin{array}{l}\text { Table S4 Percentage Composition of } \mathrm{N} 945\left[\mathrm{Ti}(\mathrm{OH})_{3}\right]_{2} \text { Frontier Molecular Orbitals } \\
(\text { (INDO/S) }\end{array}$} \\
\hline \#MO & Energy /eV & $\% \mathrm{CO}_{2}$ bpy & $\% \mathrm{Ru}$ & $\% \mathrm{NCS}$ & \%Styrylbpy \\
\hline 186 & -0.09 & 100.0 & 0.0 & 0.0 & 0.0 \\
\hline 185 & -0.56 & 71.2 & 0.0 & 0.0 & 28.7 \\
\hline 184 & -0.60 & 28.6 & 0.0 & 0.0 & 71.3 \\
\hline 183 & -1.18 & 1.0 & 1.3 & 0.1 & 97.5 \\
\hline 182 & -1.29 & 94.9 & 2.3 & 0.2 & 2.6 \\
\hline 181 & -1.55 & 20.2 & 7.9 & 1.0 & 70.9 \\
\hline $180 \mathrm{~L}$ & -1.78 & 70.2 & 6.1 & 0.8 & 22.9 \\
\hline 179H & -6.54 & 9.4 & 46.6 & 37.5 & 6.5 \\
\hline 178 & -6.82 & 8.1 & 47.6 & 41.6 & 2.7 \\
\hline 177 & -6.84 & 2.1 & 50.4 & 40.2 & 7.2 \\
\hline 176 & -7.47 & 0.2 & 0.1 & 99.5 & 0.2 \\
\hline 175 & -7.83 & 0.9 & 16.8 & 56.8 & 25.4 \\
\hline 174 & -7.91 & 8.0 & 27.5 & 61.8 & 2.6 \\
\hline 173 & -7.96 & 3.1 & 16.3 & 54.5 & 26.1 \\
\hline 172 & -8.26 & 2.4 & 7.9 & 13.5 & 76.2 \\
\hline 171 & -8.34 & 0.6 & 10.5 & 8.6 & 80.3 \\
\hline
\end{tabular}




\section{Page S6}

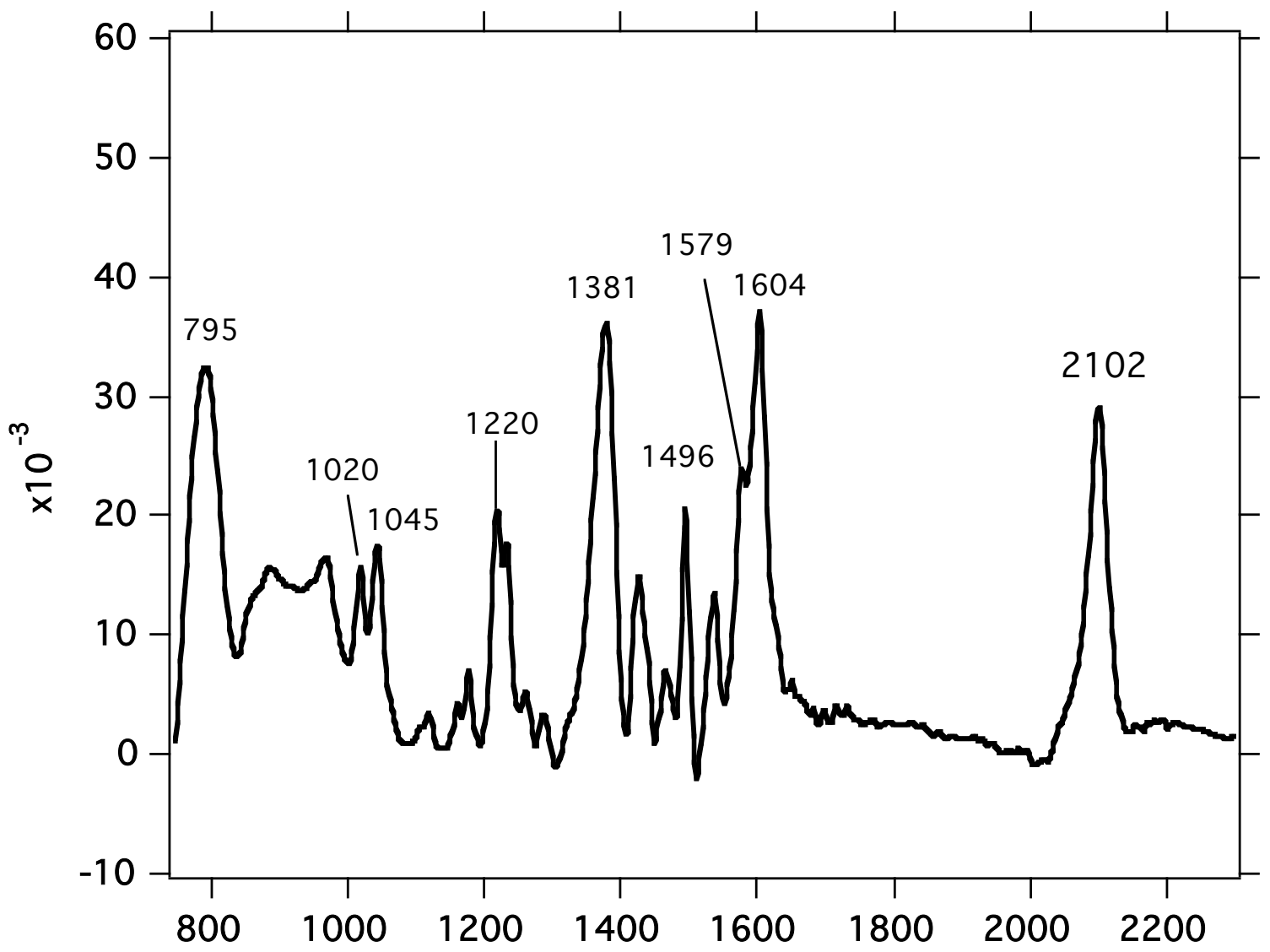

Figure S1: A portion of the ATR-FTIR spectra of the $2 \mu \mathrm{m}$ thick nanocrystalline $\mathrm{TiO}_{2}$ film anchored with N945H complex. 


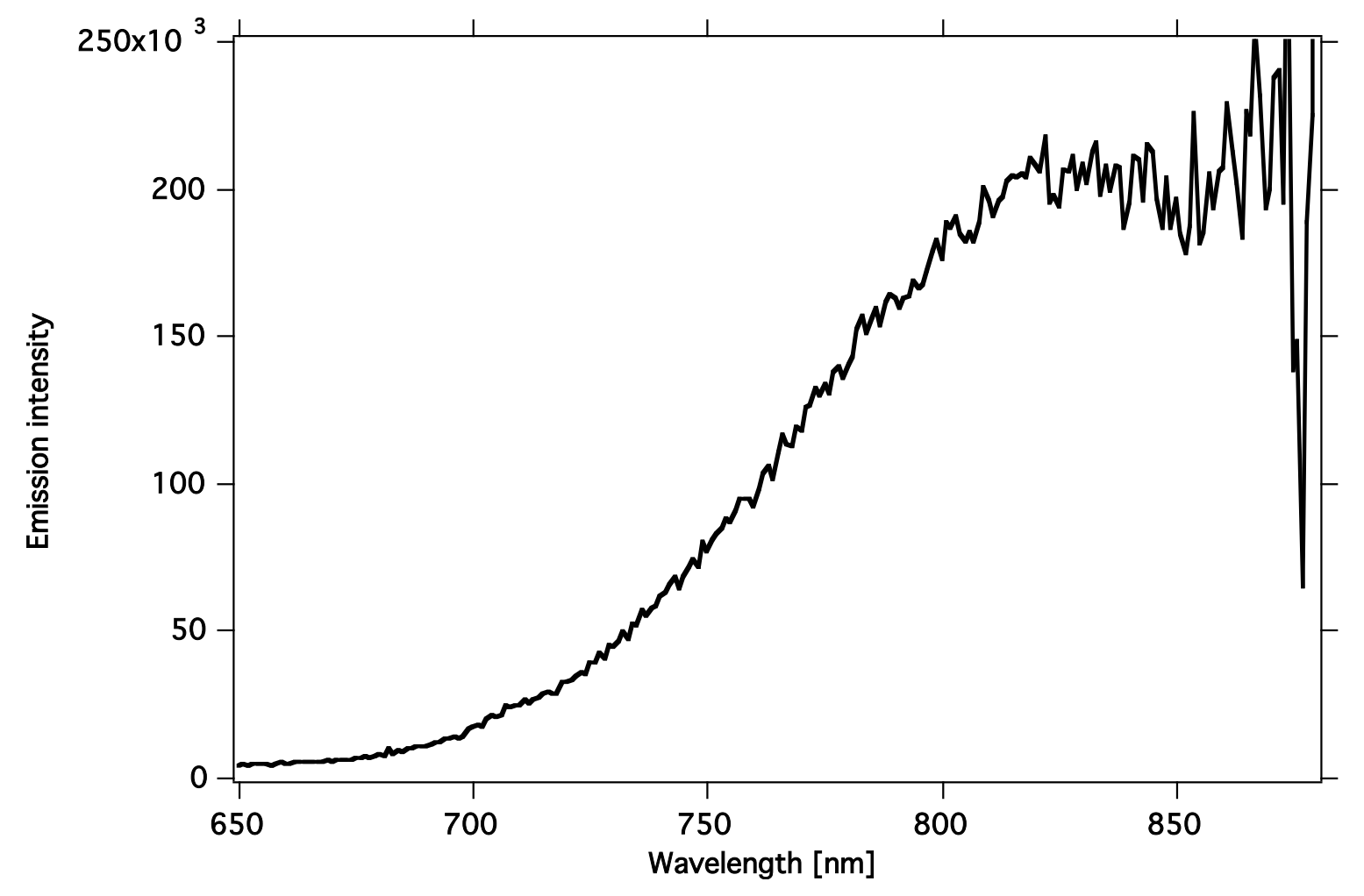

Figure S2: Emission spectra of the N945H complex measured at $298 \mathrm{~K}$ in an air-equilibrated 1:1 acetonitrile and tert-butanol solution. 